\title{
Need for a Paradigm Shift in Adult Overweight and Obesity Management - an EASO Position Statement on a Pressing public Health, Clinical and Scientific Challenge in Europe
}

\author{
Gema Frühbeck ${ }^{a, b}$ Hermann Toplak ${ }^{a, c}$ Euan Woodward ${ }^{a}$ \\ Jason C.G. Halford ${ }^{a}$ d Volkan Yumuk ${ }^{a, e}$ \\ a European Association for the Study of Obesity on behalf of the Executive Committee; \\ ${ }^{b}$ Department of Endocrinology \& Nutrition, Clínica Universidad de Navarra, University \\ of Navarra, Obesity \& Adipobiology Group of the Instituto de Investigación Sanitaria \\ de Navarra, CIBERobn, Instituto de Salud Carlos III, Pamplona, Spain; ' Department of \\ Internal Medicine, Universitäts-Klinik für Innere Medizin, Graz, Austria; ${ }^{d}$ Department of \\ Psychological Sciences, University of Liverpool, Liverpool, UK; ' Division of Endocrinology, \\ Metabolism \& Diabetes, Istanbul University Cerrahpasa Medical Faculty, Istanbul, Turkey
}

\section{Obesity, an Under-Diagnosed and Under-Treated Condition}

Recognition of the magnitude and impact of obesity as a major health problem by physicians, the general population and policy makers lies at the foundation of developing effective strategies to successfully implement medical interventions in the management of both overweight and obesity. Despite the evident physical changes imposed by excess weight, surprisingly clear opportunities for overweight and obesity diagnosis as well as treatment are being missed with approximately less than $40 \%$ of primary care physicians computing their patients' BMIs [1]. Moreover, only about one third of obese patients recall their doctor counselling them about losing weight, even though patients whose doctors discuss the problem with them are more likely to do something about it [1]. In spite of its high prevalence, obesity is perceived and documented by physicians only in a minority of affected subjects, with even around $25 \%$ of patients with excess weight being thought to be of normal weight by their primary care doctors showing that this harmful condition is notably underestimated in medical records [2-4]. Of note, hospital out-patient departments like orthopaedics, cardiology and rheumatology that receive many patients with obesity-associated co-morbidities showed an average apparent sixfold lower rate of obesity when compared to the true prevalence ( $3 \%$ vs. $25 \%$ in orthopaedics, $4 \%$ vs. $30 \%$ in cardiology, and $5 \%$ vs. $20 \%$ in rheumatology) in a retrospective analysis [5]. 
Frühbeck et al.: Need for a Paradigm Shift in Adult Overweight and Obesity

Management - an EASO Position Statement on a Pressing public Health, Clinical and

Scientific Challenge in Europe

Various possible explanations can underlie the huge disparity between apparent and true prevalence. While the need to treat the consequences of obesity (such as type 2 diabetes, hyperlipidaemia and hypertension) is readily accepted by clinicians, the disease itself is often not really tackled [6]. Some physicians may not have the skills or time to address this complex heterogeneous problem in the short consultation time available in the out-patient setting. Both overweight and obesity require a coordinated care from a multidisciplinary group combining individualised dietary advice, exercise and behavioural programmes, with some patients requiring the added support of pharmacotherapy or surgery [7]. Additionally, the paucity of specialised services dealing with overweight and obesity can also discourage referral of these patients.

\section{Clinical Inertia Accompanied by Futility and Recidivism Arguments}

Clinical inertia is the tendency to maintain current treatment strategies despite results demanding escalation. In excess weight problems this attitude substantially contributes to the disconnection between clinical aspirations for overweight/obese patients and targets achieved. In this scenario, physicians have low expectations for their patients, despite the belief of the importance of a healthy weight control through lifestyle changes and pharmacological or surgical interventions in specific cases. Conversely, overweight and obese patients sometimes do only have a rudimentary understanding of the risks of co-morbidities and the importance of their adequate control. Gaps in screening as well as in providing treatment recommendations or in performing adequate patient referral may be also signs of 'clinical inertia' implying overestimation of care provided, use of weak reasons to avoid intensification of therapy as well as lack of education and training [8, 9]. Importantly, until recently obesity has not featured strongly in medical training underlining the need to bring the topic of overweight/obesity more into the focus of the medical curriculum and for enhancing medical students' awareness of their underlying complex aetiology [8-11]. Although obesity has been declared a 21st century pandemic by the World Health Organisation some physicians in training are uncomfortable managing obesity and may even feel poorly trained to address this epidemic. Weight bias is commonly observed by students in health disciplines, who themselves report frustrations and stereotypes about treating patients with obesity [12]. Thus, students reported that patients with obesity are a common target of negative attitudes and derogatory humour by peers (63\%), health care providers (65\%), and instructors (40\%). Although $80 \%$ of students felt confident to treat obesity, many reported that patients with obesity lack motivation to make changes (33\%), had feelings of frustration (36\%) and are non-compliant with treatment (36\%) [12]. Interestingly, students with higher weight bias expressed greater frustration in these areas. The effect of students' weight bias on expectations for treatment compliance of patients with obesity was partially mediated by beliefs that obesity is caused by behavioural factors. Stigmatizing attitudes towards overweight and obesity as well as negative attitudes arise on the basis of holding the individual accountable for the excess weight [13]. One of the major barriers is the lack of specific residency education on obesity management. In this context, it would be useful to incorporate a multimodal, longitudinal obesity-specific didactic curriculum into residency programmes to improve residents' knowledge, attitudes, practice behaviours and clinical outcomes $[14,15]$ via the provision of balanced education about the controllability of obesity, focusing on the genetic, environmental and psychosocial factors as well as on the influence of lifestyle changes as regards dietary habits and physical activity together with the currently existing treatment approaches that have been proven to be effective and safe. 
Frühbeck et al.: Need for a Paradigm Shift in Adult Overweight and Obesity

Management - an EASO Position Statement on a Pressing public Health, Clinical and

Scientific Challenge in Europe

Moreover, weight bias in the health care setting appears to be a global issue [12,16-18]. Negative attitudes have been observed in medical students, qualified practitioners, nurses as well as dietitians, and may lead to beliefs about the usefulness of intervention, thus representing a barrier to good practice [16, 18-21]. Furthermore, information on weight history has been shown to impact upon the appraisal of peers with the effects being dependent on gender and BMI whereby knowing that a young female was overweight triggered negative evaluations from females but did not appear to impact the judgment of males [22]. Curiously, health professionals perceived to be overweight or obese may be vulnerable to biased attitudes from patients themselves, and physicians' excess weight may negatively affect patients' perceptions of their credibility, level of trust and inclination to follow medical advice [23]. Stronger weight bias by patients led to less trust, less compassion, less inclination to follow advice and higher inclination to change doctors when the physician was presented as obese.

Whilst plenty of people have resolved to lose weight, the perception of both the general population and health care providers may be disappointing in the sense that most of the patients give up trying after a short period of time and few will sustain the weight lost in the long term. Physicians may be negatively influenced by an abundance of reports characterised by a heavy pessimism about the long-term effectiveness of treatments overall [24]. Traditionally, two arguments have been put forward by physicians to justify passive attitudes: i) that there is nothing that can be done and ii) that the recidivism rate is too high. Lack of motivation to work with overweight and obese patients due to negative perceptions on treatment efficacy or adherence represent an evident barrier to successfully implement interventions in the health care setting. In addition, there is no ethical validity to sustain the futility and recidivism arguments. Patients suffering chronic, incurable diseases are not abandoned because we are not able to cure them, and we also do not neglect patients because the recidivism rate of certain pathologies is high. Furthermore, in clinical practice $100 \%$ patient compliance is rare. In fact, under given circumstances, efficiency of even the most efficacious treatment may be low; even the strongest and most effective antibiotic treatment will fail if not followed as prescribed by the specialist. Of note, in overweight and obesity treatment any intervention which causes a negative energy balance is guaranteed to be effective in producing weight loss. It has been estimated that affecting energy balance by only $50-100 \mathrm{kcal} / \mathrm{day}$, an easily attainable target, could prevent weight gain in most of the population [25]. Moreover, evidence-based guidelines have recognised the clinical benefits of moderate 5-10\% weight loss, which is achievable using a variety of available interventions $[7,26]$.

\section{Time for Action versus Complacency}

Obesity is a harbinger of a multitude of debilitating and fatal diseases [27]. It is clearly imperative that obesity is targeted as an area for immediate action and priority. The insidious and unsustainable image of failure in the treatment of obesity pervades and discourages many physicians. Although obesity is a particularly challenging medical condition to treat due to its complex aetiology and multifactorial nature, well-recognised effective approaches for the treatment of obesity have been identified [7, 26]. A comprehensive, pro-active, clear strategy and sustainable response to deal with the challenges posed by the overweight and obesity epidemic is urgently needed. Encouraging the instauration of programmes for early competent diagnosis, treatment and prevention is mandatory. Overweight and obesity are serious, chronic health issues that will only worsen without thoughtful and evidence-based interventions that address both the individual and the society as a whole as well as their environmental context [27]. 
Frühbeck et al.: Need for a Paradigm Shift in Adult Overweight and Obesity

Management - an EASO Position Statement on a Pressing public Health, Clinical and

Scientific Challenge in Europe

\section{What Are the Objectives of Overweight and Obesity Management?}

Who needs to lose weight? What are we trying to achieve? What are the medical benefits of weight loss? Is weight reduction always required or sufficient? Is BMI an appropriate measure? What are alternative measures? In-depth evaluation of these questions and overall benefit/risk evaluation are key issues. In this context, it should be discussed if a specific percentage weight reduction should be accepted as a primary endpoint given that several additional benefits not requiring weight loss can be of equal clinical relevance [7]. Moreover, rapid weight loss due to drastic energy restriction should not be encouraged since it usually takes place at the expense of water and the fat-free mass, therefore resulting in detrimental changes in the body composition. Improving metabolic control, cardiovascular fitness and muscle strength as well as increasing fat mass loss and preserving lean body mass, even without overall changes in final body weight, are likewise important. This approach is particularly advisable in obese elderly patients in whom treatment should focus on improvement of functional impairments, metabolic complications and obesityrelated diseases, rather than on weight loss per se. Adding exercise training to moderate energy restriction results in favourable changes to metabolism, fitness and body composition that may take place in the absence of weight loss [28]. Prevention of further weight gain constitutes an important and first step in overweight and obesity management. Weight loss should be encouraged for obese individuals suffering from pathologies related to the mechanical and/or psychosocial impact of excess weight, with an increase in physical activity according to the possibilities of each individual being included in lifestyle interventions as it offers additional benefits. Importantly, more focus should be put on a wider array of endpoints and a mere weight- or BMI-centric approach abandoned. Moreover, introduction of new criteria for identifying patients at high risk should be discussed. What are key factors that could impact outcomes? Clarification and updating of both primary and secondary endpoints going beyond specific cut-offs for BMI or relevant weight reduction including consideration of short- and long-term beneficial effects should be pursued. Weight gain represents the consequence of many interacting processes, and for a disease with such a multifactorial aetiology there will be different approaches to its management that will be more or less effective to different individuals. Thus, it is important to try to personalise management strategies as much as possible.

\section{How to Deal with the Obesity Paradox and Metabolically Healthy Obesity?}

Overweight and obesity have been classically associated with increased all-cause mortality, which is generally lowest for a BMI of $20.0-24.9 \mathrm{~kg} / \mathrm{m}^{2}$, with the risk of death conforming to a J-shaped curve $[29,30]$. However, recent meta-analyses and epidemiological studies reported that subjects with BMIs in the overweight $\left(25.0-29.9 \mathrm{~kg} / \mathrm{m}^{2}\right)$ and obesity I grade category (30.0-34.9 $\left.\mathrm{kg} / \mathrm{m}^{2}\right)$ exhibited a lower death rate than those with normal weight [31-33]. These findings led to the formulation of the 'obesity paradox' highlighting that a BMI in the excess weight range may provide a survival advantage over that in the normal range $\left(18.5-24.9 \mathrm{~kg} / \mathrm{m}^{2}\right)$. The true significance of these findings has been questioned for several reasons [34-37]. For instance, more sensitive indicators than BMI like body adiposity and its distribution should be projected on mortality to plausibly yield more accurate associations with an increased health risk. Furthermore, death postponement in the excess weight categories does not necessarily translate into a longer disease-free life. Noteworthy, an increasing BMI $>25 \mathrm{~kg} / \mathrm{m}^{2}$ confers a survival disadvantage when focusing on morbidity rather than mortality [38]. The implications on prognosis of the limitations of the BMI together with other 
Frühbeck et al:: Need for a Paradigm Shift in Adult Overweight and Obesity

Management - an EASO Position Statement on a Pressing public Health, Clinical and

Scientific Challenge in Europe

relevant influences such as aging, nutrition, physical activity, fitness, maintaining function and chronic disease in the potential protective effect of overweight/obesity should be carefully considered [39]. Further recognized sources of bias include i) reverse causation, ii) selective survival bias and iii) selection bias, all of which may be magnified in circumstances in which the studied sample is restricted to individuals suffering from serious disease or to individuals with diseases for which obesity is a cause, thereby causing methodological problems that need to be carefully considered before concluding that obesity is protective. This is of particular relevance since the obesity paradox could result in confusing messages that derail beneficial environmental changes and lead to reduced efforts by physicians to provide healthy lifestyle treatment plans to their obese patients [39].

In this same line, recently great interest has been drawn to a subgroup of individuals with obesity that does not seem to be at an increased risk for metabolic complications of obesity. This obesity sub-phenotype is being referred to as metabolically healthy obesity (MHO) [40]. It has been suggested that individuals with MHO do not significantly improve their cardiometabolic risk upon weight loss interventions and may therefore not benefit to the same extent as obese patients with metabolic co-morbidities from early lifestyle interventions, pharmacotherapy or bariatric surgery. However, it can be debated whether MHO individuals are really healthy, especially since no general agreement exists on accepted criteria to define MHO [41]. In addition, overall health of MHO individuals may be significantly impaired by several psychosocial factors, psychosomatic co-morbidities, osteoarthritis, chronic pain, diseases of the respiratory system as well as digestive and renal disorders among others. There are still open questions as to whether MHO can be considered an established phenotype or rather represents a transient stage changing with ageing, behavioural and environmental factors [38, 41, 42]. In fact, MHO appears to be a relatively transient condition with a considerable percentage of these individuals exhibiting similar metabolic derangements to those observed in obese metabolically altered patients as time passes $[43,44]$. In this line, time of exposure to the adverse effects of obesity should be of particular concern with respect to childhood obesity where cardiometabolic alterations are reportedly already evident [45-47]. Conversely, elevated concentrations of cardiometabolic risk factors have been also observed in non-obese individuals according to BMI but obese based on body fat $[48,49]$, thus showing that this sub-phenotype should be equally considered by health care professionals.

\section{Role of Pharmacotherapy}

Anti-obesity drugs have played only a limited role lately in clinical treatment algorithms for obese patients since a number of drug suspensions negatively impacted upon the obesity pharmacotherapy landscape [50]. In comparison with other diseases, at present there is a paucity of drug therapy options for obesity influenced by a history of past failures in trying to overcome the regulatory requisites of safety and efficacy. Undoubtedly, any intervention achieving a negative energy balance over an extended time period will result in weight loss. In this sense, it is important to inform patients about the rationale and beneficial effects of the different recommendations and treatment strategies. Realistic expectations about the true effect of anti-obesity drugs are needed to correctly advise patients. Pharmacological treatment of obesity is most successful if realistic goals are set, a balanced diet is stressed and a safe rate of weight loss per week is achieved through a combination of moderate caloric intake reduction (diet) and increased energy expenditure (physical activity). Poor responders or non-responders to specific drugs should be identified to discontinue pharmacological treatment with that agent, and other alternatives should be tested. 
Frühbeck et al.: Need for a Paradigm Shift in Adult Overweight and Obesity

Management - an EASO Position Statement on a Pressing public Health, Clinical and

Scientific Challenge in Europe

The advent of an increasing array of drugs, which are effective both in inducing weight loss and in maintaining weight loss achieved by lifestyle measures represent a hopeful scenario for both patients and physicians. The development of these anti-obesity agents has followed diverse paths, from lorcaserin, a selective serotonin agent, relying on the beneficial central actions of fenfluramine but without the associated systemic side-effects, to the drug combination of phentermine/topiramate, exploiting two already known drugs but using them in lower doses than with previous therapeutic uses, resulting in an additive effect on weight loss and fewer side-effects, or liraglutide, a gut hormone already applied as a glucoselowering agent but with added appetite-suppressant properties applied in a higher dose. Although anti-obesity drugs lead to clinically meaningful weight loss when prescribed as monotherapy, the use of these anti-obesity agents as adjunctive therapy post intensive lifestyle intervention could prove equally useful in weight loss maintenance. Furthermore, a progressive approach to the simultaneous use of diverse anti-obesity drugs to take advantage of different mechanisms of action (as is usually being performed in hypertension or type 2 diabetes pharmacological control) offers a further opportunity to tackle the obesity epidemic in some patients. Combination therapy will probably be useful to avoid compensatory mechanisms and potentiate initial weight loss whilst avoiding weight regain. New glucose-lowering treatments, especially glucagon-like peptide- 1 receptor agonists and sodium glucose cotransporter-2 inhibitors, offer advantages over traditional anti-diabetic drugs by improving glucose control while simultaneously promoting weight loss. Thus, improved knowledge about body weight control opens new prospects for the potential use of peptides derived from the gut or the adipose tissue. A further critical question is what constitutes an appropriate duration for a pharmacological treatment of a chronic disease? How should reimbursement of anti-obesity drugs be managed? Revisions of these requirements both with respect to efficacy and safety are warranted.

\section{Bariatric and Metabolic Surgery}

Bariatric and/or metabolic surgery should be considered for morbidly, well-informed, and motivated obese patients with previous failure to conventional treatment, who fulfil the currently established selection criteria and in whom the operative risks are acceptable [51, 52]. Candidates for surgical procedures should be selected carefully after evaluation by a comprehensive, experienced, multidisciplinary team with medical, surgical, nutritional and psychiatric expertise working in a clinical setting with adequate support for all aspects of diagnosis and management providing both pre- and post-operative counselling and support. Noteworthy, effectiveness of surgery does not necessarily need to be circumscribed to body weight alone but should also focus on the beneficial effects on co-morbidities.

\section{Taking the Patient View into Consideration}

While in many areas of medicine the participation of patients is encouraged, this has traditionally not been considered in overweight and obesity. With the paucity of available tools for physicians to combat obesity, regulatory authorities should incorporate the patient perspective when performing thorough risk/benefit analyses. Whereas numerous studies highlight the relevance of sociocultural factors influencing incidence and prevalence of obesity, only a few address how obese people perceive causes and prevention of or intervention for obesity. In this context, more attention has to be paid to the interactions of medical aspects and the social dimension of obesity [53]. 


\section{Future Perspectives}

Given the far-reaching medical, social and economic consequences of having to deal with the ill consequences and difficulties associated with obesity management, the relevance of prevention becomes evident not only for patients and health professionals but also for politicians and the society as a whole. Past and recent prevention efforts may have been hampered to some extent by a number of external factors, ranging from the lack of acceptance of obesity as a serious health problem to commercial interests. Current public health policy initiatives must be mindful of the risks of reframing obesity to inadvertently reinforce stigma, narrow responses to those aiming to modify individual behaviour and biology, and neglect population policies aiming to reduce the consumption of energy-dense foods and sedentarism. Gaining excessive body fat is a continuous, frequently progressive process. The current childhood obesity epidemic will fully manifest its deleterious consequences in future years when adolescents reach adulthood. While prevention is essential even before excess weight sets in, it is imperative to remember that moderate (5-10\%) weight loss, which is achievable using a variety of interventions, yields clinical benefits and, thus, should be encouraged in patients studied in an individualised way.

\section{Disclosure Statement}

GF has participated in a Novo Nordisk Obesity Scientific Communication Global Advisory Board Meeting. HT has served as an advisor for Vivus and Novo Nordisk. JH declares his involvement as a member of a U.K. Novo Nordisk Advisory Board.

\section{References}

1 Frühbeck G: Obesity. Screening for the evident in obesity. Nat Rev Endocrinol 2012;8:570-572.

-2 Hauner H, Koster I.,von Ferber L: Frequency of 'obesity' in medical records and utilization of out-patient health care by 'obese' subjects in Germany. An analysis of health insurance data. Int J Obes Relat Metab Disord 1996; 20:820-824.

3 Caccamese SM, Kolodner,K, Wright SM: Comparing patient and physician perception of weight status with body mass index. Am J Med 2002;112:662-666.

4 Ferraro KF, Holland KB: Physician evaluation of obesity in health surveys: 'who are you calling fat?'. Soc Sc Med 2002;55:1401-1413.

-5 Cleator J, Richman E, Leong KS, Mawdsley L, White S, Wilding J; Obesity: under-diagnosed and under-treated in hospital outpatient departments. Int J Obes Relat Metab Disord. 2002;26:581-584.

6 Frühbeck G, Diez-Caballero A, Gómez-Ambrosi J, Cienfuegos JA, Salvador J: Preventing obesity. Doctors underestimate obesity. BMJ 2003;326:102-103.

7 Yumuk V, Frühbeck G, Oppert JM, Woodward E, Toplak H: An EASO position statement on multidisciplinary obesity management in adults. Obes Facts 2014;7:96-101.

8 Frühbeck G: Is Europe really battling obesity? Obes Facts 2010;3:219-221.

- Hall JI: Obesity - a reluctance to treat? Obes Facts 2010;3:79-80.

10 Banasiak M, Murr MM: Medical school curricula do not address obesity as a disease. Obes Surg 2001;11:677679.

11 Swift JA, Sheard C, Rutherford M: Trainee health care professionals' knowledge of the health risks associated with obesity. J Hum Nutr Diet 2007;20:599-604.

12 Puhl RM, Luedicke J, Grilo CM: Obesity bias in training: attitudes, beliefs, and observations among advanced trainees in professional health disciplines. Obesity (Silver Spring) 2014;22:1008-1015.

-13 Pantenburg B, Sikorski C, Luppa M, Schomerus G, König HH, Werner P, Riedel-Heller SG: Medical students' attitudes towards overweight and obesity. PLoS ONE 2012;7:e48113.

14 Acosta A, Azzalin A, Emmons CJ, Shuster JJ, Jay M, Lo MC: Improving residents' clinical approach to obesity: impact of a multidisciplinary didactic curriculum. Postgrad Med J 2014;90:630-637.

15 Kushner RF, Zeiss DM, Feinglass JM, Yelen M: An obesity educational intervention for medical students addressing weight bias and communication skills using standardized patients. BMC Med Educ 2014;14:53. 
Frühbeck et al.: Need for a Paradigm Shift in Adult Overweight and Obesity

Management - an EASO Position Statement on a Pressing public Health, Clinical and Scientific Challenge in Europe

16 Harvey EL, Glenny A, Kirk SF, Summerbell CD: Improving health professionals' management and the organisation of care for overweight and obese people. Cochrane Database Syst Rev 2001;2:CD000984.

17 Harvey EL, Hill AJ: Health professionals' views of overweight people and smokers. Int J Obes Relat Metab Disord 2001;25:1253-1261.

18 Harvey EL, Summerbell CD, Kirk SF, Hill AJ: Dietitians' views of overweight and obese people and reported management practices. J Hum Nutr Diet 2002;15:331-347.

19 Teachman BA, Brownell KD: Implicit anti-fat bias among health professionals: is anyone immune? Int J Obes Relat Metab Disord 2001;25:1525-1531.

20 Teachman BA, Gapinski KD, Brownell KD, Rawlins M, Jeyaram S: Demonstrations of implicit anti-fat bias: the impact of providing causal information and evoking empathy. Health Psychol 2003;22:68-78.

-21 Foster GD, Wadden TA, Makris AP, Davidson D, Sanderson RS, Allison DB, Kessler A: Primary care physicians' attitudes about obesity and its treatment. Obes Res 2003;11:1168-1177.

22 Tucci SA, Boyland EJ, Halford JC, Harrold JA: Stigmatisation of a formerly obese young female. Obes Facts 2013; 6:433-442.

23 Puhl RM, Gold JA, Luedicke J, DePierre JA: The effect of physicians' body weight on patient attitudes: implications for physician selection, trust and adherence to medical advice. Int J Obes (Lond) 2013;37:1415-1421.

24 Harvey EL, Glenny AM, Kirk SF, Summerbell CD: An updated systematic review of interventions to improve health professionals' management of obesity. Obes Rev 2002;3:45-55.

25 Hill JO: Understanding and addressing the epidemic of obesity: an energy balance perspective. Endocr Rev 2006;27:750-761.

26 Hill JO, Peters JC, Catenacci VA, Wyatt HR: International strategies to address obesity. Obes Rev 2008;9(suppl 1):41-47.

27 Frühbeck G, Toplak H, Woodward E, Yumuk V, Maislos M, Oppert JM; Executive Committee of the European Association for the Study of Obesity: Obesity: the gateway to ill health - an EASO position statement on a rising public health, clinical and scientific challenge in Europe. Obes Facts 2013;6:117-120.

-28 Miller CT, Fraser SF, Levinger I, Straznicky NE, Dixon JB, Reynolds J, Selig SE: The effects of exercise training in addition to energy restriction on functional capacities and body composition in obese adults during weight loss: a systematic review. PLoS ONE 2013;8:e81692.

29 Calle EE, Thun MJ, Petrelli JM, Rodriguez C, Heath CW: Body-mass index and mortality in a prospective cohort of U. S. adults. N Engl J Med 1999;341:1097-1105.

-30 Berrington de Gonzalez A, Hartge P, Cerhan JR, Flint AJ, Hannan L, MacInnis RJ, Moore SC, Tobias GS, AntonCulver H, Freeman LB, Beeson WL, Clipp SL, English DR, Folsom AR, Freedman DM, Giles G, Hakansson N, Henderson KD, Hoffman-Bolton J, Hoppin JA, Koenig KL, Lee IM, Linet MS, Park Y, Pocobelli G, Schatzkin A, Sesso HD, Weiderpass E, Willcox BJ, Wolk A, Zeleniuch-Jacquotte A, Willett WC, Thun MJ: Body-mass index and mortality among 1.46 million white adults. N Engl J Med 2010;363:2211-2219.

-31 Carnethon MR, De Chavez PJ, Biggs ML, Lewis CE, Pankow JS, Bertoni AG, Golden SH, Liu K, Mukamal KJ, Campbell-Jenkins B, Dyer A: Association of weight status with mortality in adults with incident diabetes. JAMA 2012;308:581-90.

-32 Flegal KM, Kit BK, Orpana H, Graubard BI: Association of all-cause mortality with overweight and obesity using standard body mass index categories: a systematic review and meta-analysis. JAMA 2013;309:71-82.

-33 Lavie CJ, De Schutter A, Milani RV: Healthy obese versus unhealthy lean: the obesity paradox. Nat Rev Endocrinol 2014; doi: 10.1038/nrendo.2014.165.

34 Heymsfield SB, Cefalu WT: Does body mass index adequately convey a patient's mortality risk? JAMA 2013; 309:87-88.

-35 Camhi SM, Katzmarzyk PT: Differences in body composition between metabolically healthy obese and metabolically abnormal obese adults. Int J Obes (Lond) 2014;38:1142-1145.

-36 Aung K, Lorenzo C, Hinojosa MA, Haffner SM: Risk of developing diabetes and cardiovascular disease in metabolically unhealthy normal-weight and metabolically healthy obese individuals. J Clin Endocrinol Metab 2014; 99:462-468.

-37 Ahima RS, Lazar MA: Physiology. The health risk of obesity - better metrics imperative. Science 2013;341: 856-858.

-38 Blundell JE, Dulloo AG, Salvador J, Frühbeck G; on behalf of the EASO SAB Working Group on BMI: Beyond BMI - phenotyping the obesities. Obes Facts 2014;7:322-328.

-39 Stevens J, Bradshaw PT, Truesdale KP, Jensen MD: Obesity paradox should not interfere with public health efforts. Int J Obes (Lond) 2014: doi: 10.1038/ijo.2014.60.

40 Stefan N, Häring H-U, Hu FB, Schulze MB: Metabolically healthy obesity: epidemiology, mechanisms, and clinical implications. Lancet Diabetes Endocrinol 2013;1:152-162.

-41 Kramer CK, Zinman B, Retnakaran R: Are metabolically healthy overweight and obesity benign conditions? A systematic review and meta-analysis. Ann Intern Med 2013;159:758-69.

42 Hill JO, Wyatt HR: The myth of healthy obesity. Ann Intern Med 2013;159:789-790.

43 Chang, Y, Ryu S, Suh BS, Yun KE, Kim CW, Cho SI: Impact of BMI on the incidence of metabolic abnormalities in metabolically healthy men. Int J Obes (Lond) 2012;36:1187-1194.

-44 Gómez-Ambrosi J, Catalán V, Rodríguez A, Andrada P, Ramírez B, Ibáñez P, Vila N, Romero S, Margall MA, Gil MJ, Moncada R, Valentí V, Silva C, Salvador J, Frühbeck G: Increased cardiometabolic risk factors and inflammation in adipose tissue in obese subjects classified as metabolically healthy. Diabetes Care 2014;37:2813-2821. 
Frühbeck et al.: Need for a Paradigm Shift in Adult Overweight and Obesity

Management - an EASO Position Statement on a Pressing public Health, Clinical and

Scientific Challenge in Europe

-45 Cote AT, Harris KC, Panagiotopoulos C, Sandor GG, Devlin AM: Childhood obesity and cardiovascular dysfunction. J Am Coll Cardiol 2013;62:1309-1319.

-46 Reis JP, Hankinson AL, Loria CM, Lewis CE, Powell-Wiley T, Wei GS, Liu K: Duration of abdominal obesity beginning in young adulthood and incident diabetes through middle age: the CARDIA study. Diabetes Care 2013;36:1241-1247.

-47 Fintini D, Chinali M, Cafiero G, Esposito C, Giordano U, Turchetta A, Pescosolido S, Pongiglione G, Nobili V: Early left ventricular abnormality/dysfunction in obese children affected by NAFLD. Nutr Metab Cardiovasc Dis 2014;24:72-74.

-48 Gómez-Ambrosi J, Silva C, Galofré JC, Escalada J, Santos S, Gil MJ, Valentí V, Rotellar F, Ramírez B, Salvador J, Frühbeck G: Body adiposity and type 2 diabetes: Increased risk with a high body fat percentage even having a normal BMI. Obesity (Silver Spring) 2011;19:1439-1444.

49 Gómez-Ambrosi J, Silva C, Galofré JC, Escalada J, Santos S, Millán D, Vila N, Ibañez P, Gil MJ, Valentí V, Rotellar F, Ramírez B, Salvador J, Frühbeck G: Body mass index classification misses subjects with increased cardiometabolic risk factors related to elevated adiposity. Int J Obes (Lond) 2012;36:286-294.

50 Manning S, Pucci A, Finer N: Pharmacotherapy for obesity: novel agents and paradigms. Ther Adv Chronic Dis 2014;5:135-148.

-51 Fried M, Yumuk V, Oppert JM, Scopinaro N, Torres AJ, Weiner R, Yashkov Y, Frühbeck G; European Association for the Study of Obesity; International Federation for the Surgery of Obesity - European Chapter: Interdisciplinary European guidelines on metabolic and bariatric surgery. Obes Facts 2013;6:449-468.

52 Fried M, Yumuk V, Oppert JM, Scopinaro N, Torres A, Weiner R, Yashkov Y, Frühbeck G; International Federation for Surgery of Obesity and Metabolic Disorders-European Chapter (IFSO-EC); European Association for the Study of Obesity (EASO); European Association for the Study of Obesity Obesity Management Task Force (EASO OMTF): Interdisciplinary European guidelines on metabolic and bariatric surgery. Obes Surg 2014;24: 42-55.

53 Braun M, Schell J, Siegfried W, Muller MJ, Ried J: Re-entering obesity prevention: a qualitative-empirical inquiry into the subjective aetiology of extreme obese adolescents. BMC Public Health 2014;14:977. 